\title{
Hemangioendotelioma epitelióide hepático: relato de caso com tratamento multimodal
}

\author{
Epithelioid hemangioendothelioma of the liver: report of a case with multimodal \\ treatment
}

Carlos Eduardo Rodrigues Santos' ${ }^{1}$ Mauro Monteiro Correia', Rodrigo da Silva Pereira², Ivanir Martins ${ }^{3}$, Cristina Moreira do Nascimento ${ }^{3}$, Lovise Dias Gruezo ${ }^{2}$, Rodrigo Mendes de Carvalho², Jurandir de Almeida Dias'

\section{Resumo}

Os autores apresentam um caso raro de hemangioendotelioma epitelióide hepático multicêntrico de uma paciente oligossintomática, tratada inicialmente com ressecção e alcoolização. Na sua recidiva, foi submetida à radioablação. Revisando a literatura, os autores concluem que a ressecção permanece como o principal tratamento, associada ou não a métodos ablativos, e que o transplante tem indicação na doença multinodular bilateral.

Palavras-chave: Hemangioendotelioma epitelióide; Neoplasia hepática; Transplante hepático; Fígado

${ }^{1}$ Seção de Cirurgia Abdominopélvica do Instituto Nacional de Câncer - INCA (RJ)

${ }^{2}$ Internato em Medicina da Universidade do Grande Rio - UNIGRANRIO (RJ)

${ }^{3}$ Serviço de Patologia do Instituto Nacional de Câncer - INCA (RJ)

Endereço para corrrespondência: Carlos Eduardo Rodrigues Santos. Praça Cruz Vermelha, 23 5o andar - Centro - Rio de Janeiro (RJ). CEP: $20230-130$.

E-mail: carloseduardo@cirurgiaonline.com.br 


\section{INTRODUÇÃO}

O hemangioendotelioma epitelióide é uma neoplasia recentemente descrita. Dail e Liebow, em 1975, fizeram a primeira descrição deste tumor no pulmão, chamandoo de "tumor bronquioloalveolar intravascular"1. A origem vascular desta neoplasia não havia sido reconhecida até $1979^{2}$, e, desde então, está sendo descrita nos seguintes órgãos: fígado, baço, osso, cérebro, meninges, mama, coração, cabeça e pescoço, partes moles, trato gastrintestinal, linfonodos e veias. Em 1982, Weiss e Enzinger descreveram pela primeira vez o hemangioendotelioma epitelióide hepático (HEH), como entidade distinta, com potencial maligno imprevisível ${ }^{3}$.

Ainda que mais de 200 casos desse tumor tenham sido relatados na literatura até o momento, muitos aspectos permanecem desconhecidos ${ }^{4}$. Além disso, sua real incidência não é fidedigna, pois tais tumores têm sido erroneamente diagnosticados como carcinomas metastáticos, colangiocarcinoma, carcinoma hepatocelular ou angiossarcoma, dentre outros 5 .

$\mathrm{O} \mathrm{HEH}$ é um tumor vascular muito raro, sendo constituído de uma mistura de células fusiformes e de células epitelióides volumosas que infiltram sinusóides e vasos sanguíneos associados à intensa reação fibrosa ${ }^{6-11}$. Varia em tamanho de focos microscópicos até tumores volumosos, medindo mais de $15 \mathrm{~cm}$. Os tumores solitários podem envolver um lobo inteiro e circundar veias porta e hepática. A imuno-histoquímica revela comumente reatividade para Fator VIII, CD34, CD31 e Vimentina ${ }^{7,12}$.

Geralmente ocorre em mulheres adultas e se apresenta como nódulos múltiplos e de distribuição predominantemente periférica ${ }^{13}$. Nenhum fator genético ou ambiental é reconhecido, porém associações com o uso de estrógenos, hormônios esteróides e colelitíase têm sido descritas $s^{7,14}$.

É caracterizado por um crescimento lento e progressivo, que pode levar à insuficiência hepática e à morte; porém, regressão espontânea do tumor também pode ser observada em alguns casos ${ }^{7}$. O prognóstico é extremamente variável, com relatos de sobrevida em cinco anos de $30 \%$, sem qualquer tratamento ${ }^{8}$.

Apesar da grande variedade de opçôes de tratamentos descritos na literatura como quimioterapia arterial e sistêmica $^{8,15}$, embolização e alcoolização ${ }^{15,16}$, a abordagem cirúrgica permanece como a melhor opção terapêutica, indo da ressecção ao transplante ${ }^{14,17}$.

$\mathrm{O} \mathrm{HEH}$ pode mimetizar tumores vasculares como o angiossarcoma ${ }^{10,11}$, mas a preservação da estrutura acinar hepática, a esclerose densa, a hialinização e a calcificação dos nódulos tumorais típicos do HEH não são observados no angiossarcoma ${ }^{7,14}$. Outros diagnósticos diferenciais são feitos com colangiocarcinoma, hepatocarcinoma fibrolamelar, sarcoma ou tumores mistos, além de carcinomas metastáticos ${ }^{3,14,18}$.

Segundo Lauffer et al., o prognóstico do HEH é melhor do que outros tumores malignos hepáticos ${ }^{7,14}$.

\section{RELATO DO CASO}

Paciente feminina, 47 anos, matriculada na Seção de Cirurgia Abdominopélvica do INCA-MS / Brasil, em março de 2000, com uma história de aumento do volume abdominal e massa palpável no hipocôndrio direito, desde janeiro de 2000. Ao exame físico apresentava-se obesa, em bom estado geral, com massa localizada em região epigástrica, de consistência endurecida, fixa e indolor, superfície irregular e bordas imprecisas. Uma tomografia computadorizada (Figura 1) demonstrou lesão heterogênea no fígado com contornos lobulados, medindo $16,3 \mathrm{~cm} \mathrm{x}$ $13,4 \mathrm{~cm}$, comprometendo os segmentos II e III, determinando rechaço do estômago, uma segunda massa no segmento $\mathrm{V}$ e colelitíase.

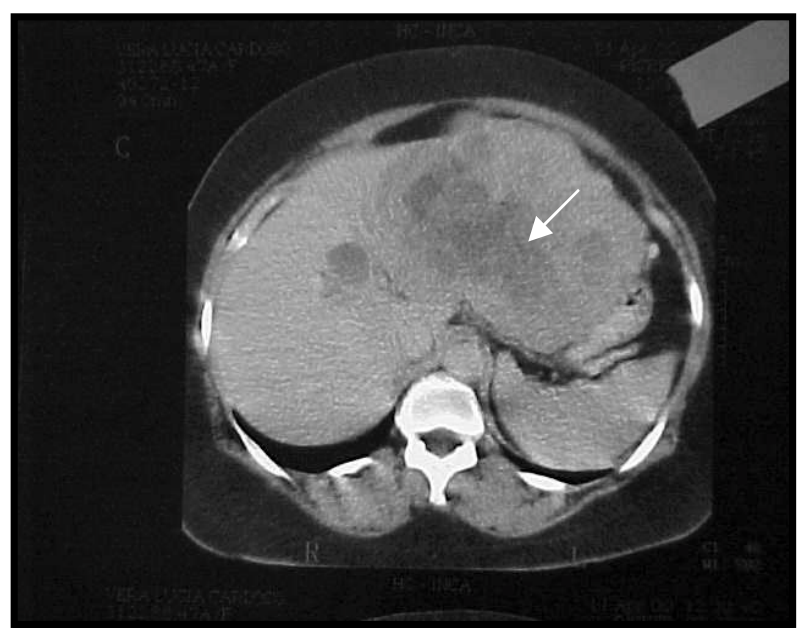

Figura 1. Imagem de tomografia computadorizada. A seta mostra volumoso tumor do lobo esquerdo do fígado.

Fonte: Serviço de Cirurgia Abdominopélvica do INCA, 2000

Os exames laboratoriais e as provas de função hepática não mostravam alterações significativas. Antígeno cárcinoembriogênico $=1,35 \mathrm{ng} / \mathrm{ml}$; alfafetoproteína $=1,81 \mathrm{U} / \mathrm{ml}$, marcadores das hepatites negativos, endoscopia digestiva alta demonstrando compressão extrínseca do estômago e duodeno; clister opaco normal. Seu risco cirúrgico foi classificado como ASA (American Society of Anesthesiologists) II, classe funcional ${ }^{\mathrm{II}}$.

Em abril foi submetida à laparotomia exploradora 
com vistas à lobectomia esquerda mais segmentectomia do V. Durante a laparotomia, foi observada massa medindo $16 \mathrm{~cm} \times 14 \mathrm{~cm}$ no lobo esquerdo e outro nódulo medindo $5,5 \mathrm{~cm} \times 3,7 \mathrm{~cm}$, ocupando o segmento $\mathrm{V}$ (Figura 2). Ao inventário, não foram vistos sinais de disseminação celômica ou outros tumores que fizessem suspeitar de que se tratasse de lesões metastáticas.

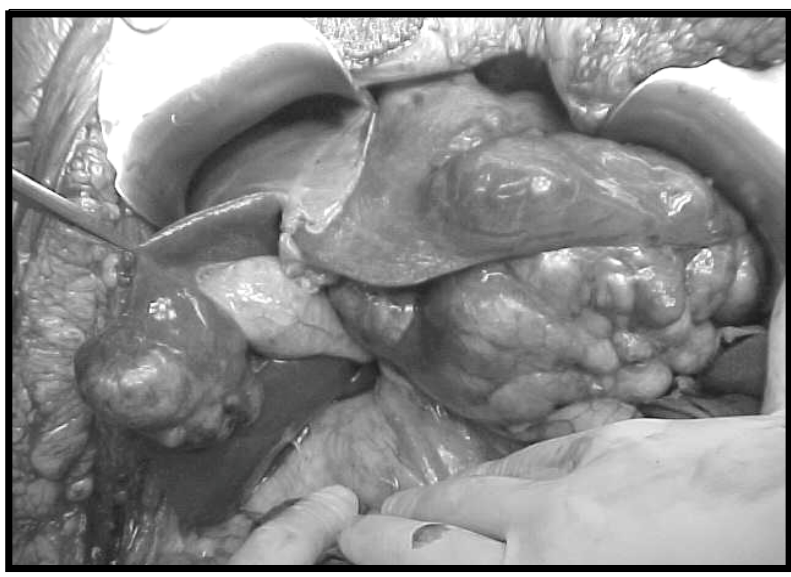

Figura 2. Aspecto intra-operatório dos tumores no lobo esquerdo e no segmento $\mathrm{V}$.

Fonte: Serviço de Cirurgia Abdominopélvica do INCA, 2000

Realizou-se ultra-sonografia intra-operatória que evidenciou um terceiro nódulo, medindo $1 \mathrm{~cm}$, entre os segmentos VIII e IVa circundado pela bifurcação dos ramos setoriais direitos e a veia supra-hepática média.

Decidiu-se começar pela colecistectomia, ressecção e exame de congelação do nódulo do segmento $\mathrm{V}$ para diagnóstico. O laudo de congelação foi de hepatocarcinoma.

Foi dado prosseguimento ao ato cirúrgico, sendo realizada então uma lobectomia esquerda (Figura 3) e punção aspirativa com agulha fina (PAAF) do nódulo do segmento VIII compatível com neoplasia maligna.

Devido à localização central do nódulo do segmento VIII, que implicaria uma trissegmentectomia esquerda e o mau prognóstico de um possível carcinoma hepatocelular multifocal, e na ausência de radioablação na ocasião, optou-se pela alcoolização deste nódulo, como proposto por alguns autores ${ }^{16}$.

Ambos os tumores foram diagnosticados na parafina como hemangioendotelioma epitelióide hepático com áreas de proliferação de células epitelióides, contendo luzes vasculares primitivas (Figura 4), grandes vacúolos claros citoplasmáticos correspondentes a lúmens vasculares primitivos (Figura 5), células epitelióides e lúmens vasculares contendo hemácias (Figura 6), infiltração da cápsula adjacente e índice mitótico de nove mitoses por 50 campos de grande aumento. $\mathrm{O}$ estudo imuno-histoquímico mostrou reatividade para Vimentina. A inclusão em parafina do nódulo do segmento VIII foi positivo para células malignas.

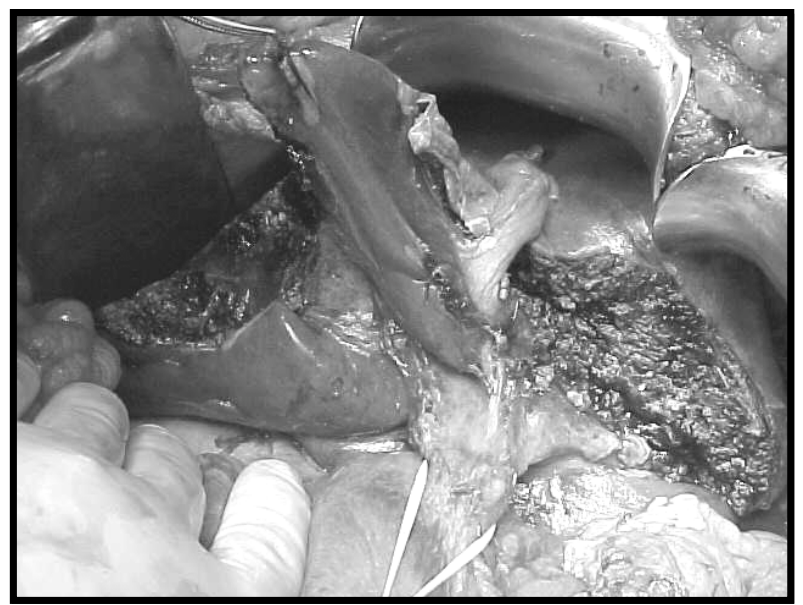

Figura 3. Aspecto intra-operatório do fígado após a ressecção. Fonte: Serviço de Cirurgia Abdominopélvica do INCA, 2000

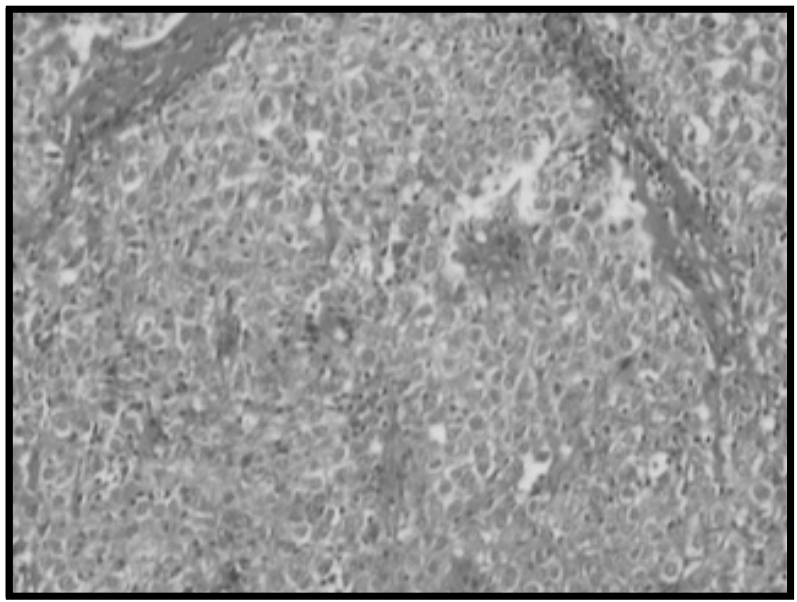

Figura 4. HEH com áreas de proliferação de células epitelióides, contendo luzes vasculares primitivas (X100, HE).

Fonte: DIPAT (INCA), 2000

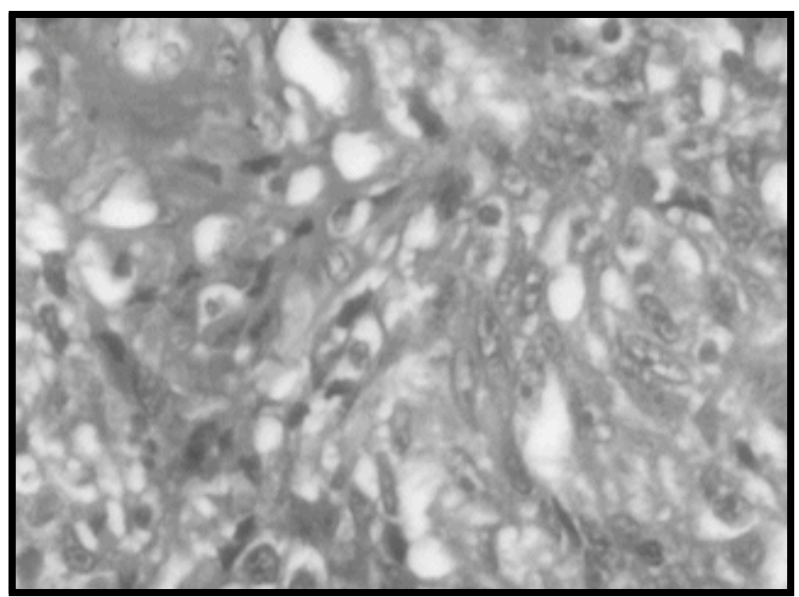

Figura 5. Grandes vacúolos claros citoplasmáticos correspondendo a lúmens vasculares primitivos (X400, HE). Fonte: DIPAT (INCA), 2000 


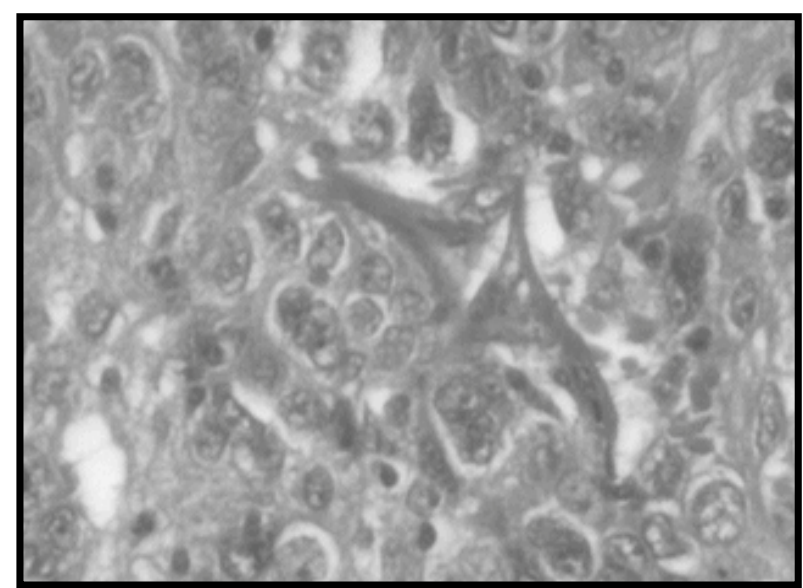

Figura 6. HEE exibindo células epitelióides e lúmens vasculares contendo hemácias (X400, HE ).

Fonte: DIPAT (INCA), 2000

A paciente apresentou uma evolução pós-operatória satisfatória, sem complicações, obtendo alta hospitalar no oitavo dia de pós-operatório. Permaneceu bem, em controle, até que no sexto mês de acompanhamento, a tomografia computadorizada evidenciou a presença de nódulos hipoecóicos nos segmentos VI e VII e crescimento do nódulo no segmento VIII. Ela foi reoperada e submetida à ablação com radiofreqüência das lesões (Figura 7). No terceiro ano e sexto mês de seguimento após a laparotomia exploradora, a paciente encontrava-se com evidência de progressão de doença hepática e peri-hepática.

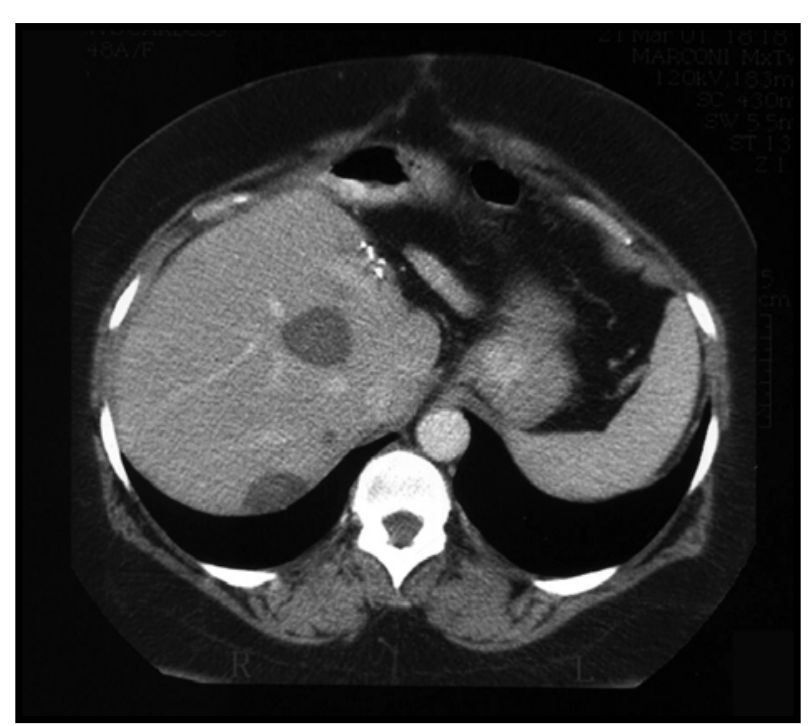

Figura 7. Tomografia computadorizada no $10^{\circ}$ mês póslaparotomia exploradora.

Fonte: Serviço de Cirurgia Abdominopélvica do INCA, 2000

\section{DISCUSSÃO}

No presente caso, as principais características epidemiológicas relativas a esta doença estiveram presentes, mas não foram observadas associações com o uso de hormônios ${ }^{7,14}$.

Clinicamente a paciente apresentava-se oligossintomática, sendo a presença de massa em epigástrio a sua única queixa, o que vem a corroborar a inespecificidade do quadro clínico. Icterícia, febre e sangramento digestivo (devido à hipertensão portal) podem estar presentes, porém são incomuns $s^{7,9,14}$. Laboratorialmente, não foram observadas alteraçōes específicas, como é descrito na literatura ${ }^{6-9,14}$. Neste caso, a única alteração observada foi uma discreta elevação do LDH.

O HEH é caracteristicamente visto, em exames de imagem, como áreas nodulares hipodensas, distribuídas difusamente no fígado, sendo cerca de $80 \%$ bilaterais, como no caso relatado ${ }^{14,18}$. Calcificação e retração capsular também são vistas freqüentemente nos achados radiológicos ${ }^{19}$.

Associações com outras doenças não são comuns, o que comprova a presente descrição de caso. Foram relatados dois casos de associação com a Síndrome de Kasabach-Merrit, que é caracterizada por hemangiomas congênitos subcutâneos e hemangiomas viscerais, trombocitopenia e coagulação intravascular disseminada ${ }^{20,21}$.

Atualmente, uma série de tratamentos vem sendo proposta para esta doença. Desde a simples observação com taxas de sobrevida de $30 \%$ a $54 \%$, em cinco anos ${ }^{7,8}$, até o transplante, passando pela quimioterapia sistêmica e intra-arterial, utilizando 5-fluorouracil e adriamicina, com resposta completa e adequado controle da doença em alguns casos ${ }^{8}$.

Foi relatado recentemente o primeiro caso de tratamento bem sucedido com o uso de interferon alfa$2 \mathrm{~B}$ combinado com ressecção hepática multifocal. $\mathrm{O}$ tratamento seguiu o protocolo de doses diárias subcutâneas de interferon alfa-2B (três milhōes de unidades) nove semanas antes e uma semana depois da ressecção dos segmentos VI e VII e subsegmentectomia do IVb com margens cirúrgicas livres. Não foi necessária transfusão sanguínea. A sobrevida livre de doença é de três anos e a paciente encontra-se assintomática. A terapia com interferon tem sido proposta para redução do tumor e prevenção das metástases ${ }^{18,22}$.

Entretanto é consenso que o tratamento cirúrgico permanece como a primeira opção sempre que 
possível $^{6,7,9,14}$, representando uma das poucas indicações de transplante hepático na terapêutica de neoplasias. Makhlouf et al. relataram, recentemente, uma série de 137 casos, em que foram utilizadas pelo menos 10 modalidades diferentes de tratamento. Os pacientes tratados cirurgicamente apresentaram taxa de sobrevida de $71,4 \%$, com segmento variando de 36 meses a 10 anos. Nessa série, o tratamento cirúrgico foi restrito ao transplante hepático. Isso se deve ao fato de, na maioria dos casos, as lesōes serem multifocais. Apenas $11 \%$ a $18 \%$ dos casos apresentam-se como nódulos únicos, sendo múltiplos em cerca $66,6 \%$ e bilaterais em $82 \%$ das vezes ${ }^{7,14}$. Esse tipo de apresentação dificulta a realização das hepatectomias.

Entretanto há concordância entre os autores de que o transplante só deve ser indicado quando todas as lesōes não puderem ser removidas pela cirurgia convencional de ressecção $0^{9,14}$. Marino et al. relatam, em sua série de transplantes, uma sobrevida de $76 \%$ em cinco anos, sendo que a presença de doença metastática não contraindicaria o tratamento ${ }^{6}$.

A dificuldade de diagnóstico histológico é um outro empecilho. O HEH é freqüentemente confundido com angiossarcoma e, nos casos relativos ao fígado, também pode haver confusão com colangiocarcinoma, carcinoma hepatocelular fibrolamelar, sarcomas ou tumores mixóides, sendo a necessidade de estudo imunohistoquímico um fator limitante do diagnóstico histológico por congelação ${ }^{7,8,12,23}$, explicando o erro diagnóstico de congelação no presente caso. Entretanto, não houve prejuízo em relação ao tratamento da paciente, já que o diagnóstico da congelação foi de uma neoplasia maligna (hepatocarcinoma) e a conduta cirúrgica seria a mesma se o diagnóstico tivesse sido de HEH.

O tumor pode ser controlado com o tratamento multimodal ${ }^{6-11,14,16-18}$, mas a evolução do presente caso com o surgimento de novos nódulos caracteriza comportamento de prognóstico reservado.

\section{CONCLUSÃO}

Apesar de existirem inúmeras possibilidades terapêuticas para o tratamento do $\mathrm{HEH}$, o tratamento cirúrgico permanece como a primeira opção ${ }^{24}$. Entretanto, as ressecções atípicas associadas a métodos alternativos como a alcoolização hepática e ablação constituem eventualmente uma forma segura e eficaz para o tratamento desta doença ${ }^{16}$.

Atualmente, para se definir a conduta ideal, cada caso deve ser analisado individualmente, lembrando-se que a ressecção representa a oportunidade real de cura, estando o transplante indicado quando toda a doença não puder ser removida senão por hepatectomia total.

\section{REFERÊNCIAS}

1. Dail DH, Liebow AA. Intravascular bronchioalveolar tumor. Am J Pathol. 1975;78:6a-7a.

2. Corrin B, Manners B, Millard M, Weaver L. Histogenesis of the so-called "intravascular bronchioloalveolar tumor." J Pathol. 1979;128(3):163-67.

3. Weiss SW, Enzinger FM. Epithelioid hemangioendothelioma: a vascular tumor often mistaken for a carcinoma. Cancer. 1982;50(5):970-81.

4. d'Annibale M, Piovanello P, Carlini P, Del Nonno F, Sciarretta F, Rossi M, et al. Epithelioid hemangioendothelioma of the liver: case report and review of the literature. Transplant Proc. 2002;34(4):1248-251.

5. Botella AG, Valladares LD, Antona EM, Pernaute AS, Aguirre EP, Ortega L, et al. Epithelioid hemangioendothelioma of the liver. J Hepatobiliary Pancreat Surg. 2006;13(2):167-71.

6. Marino IR, Todo S, Tzakis AG, Klintmalm G, Kelleher M, Iwatsuki $S$, et al. Treatment of hepatic epithelioid hemangioendothelioma with liver transplantion. Cancer. 1988;62:2079-2084.

7. Makhlouf HR, Ishak KG, Goodman ZD. Epithelioid hemangioendothelioma of the liver. A clinicopathologic study of 137 cases. Cancer. 1999;85(3):562-80.

8. Idilman R, Dokmeci A, Beyler AR, Bastemir M, Ormeci N, Aras N, et al. Successful medical treatment of epithelioid hemangioendothelioma of liver. Oncology. 1997;54:171-75.

9. Pokharna RK, Garg PK, Gupta SD, Dutta U, Tandon RK. Primary epithelioid haemangioendothelioma of the liver: case report and review of the literature. J Clin Pathol. 1997;50:1029-1031.

10. Dietze O, Davies SE, Williams R, Portmann B. Malignant epithelioid haemangioendothelioma of the liver: a clinicopathological and histochemical study of 12 cases. Histopathology. 1989;15:225-37.

11. Ishak KG, Sesterhenn IA, Goodman ZD, Rabin L, Stromeyer FW. Epithelioid hemangioendothelioma of the liver: A clinicopathologic and follow-up study of 32 cases. Hum Pathol. 1984;15:839-52.

12. Demetris AJ, Minervini M, Raikow RB, Lee RG. Hepatic epithelioid hemangioendothelioma biological questions based on pattern of recurrence in an allograft and immunophenotype. Am J Surg Pathol. 1997;21(3):263-70.

13. Miller WJ, Dodd GD, Federle MP, Baron RL. Epithelioid hemangioendothelioma of the liver: imaging findings with pathologic correlation. AJR Am J Roentgenol. 1992;159:53-57.

14. Lauffer JM, Zimmermann A, Krahenbuhl L, Triller J, Baer HU. Epithelioid hemangioendothelioma of liver. A rare 
hepatic tumor. Cancer. 1996;78(11):2318-326.

15. Kawabe T, Tagawa K, Unuma T, Takanashi R, Oka T. Hepatic epithelioid hemangioendothelioma in a young female. Dig Dis Sci. 1987;32(12):1422-427.

16. Shiro Y, Nakamura U, Hashiguchi M, Nomiyama K, Nagamatsu A, Akagi K, et al. Case of liver epithelioid hemangioendothelioma treated with percutaneous ethanol infusion. Nippon Naika Gakkai Zasshi. 1996;85(6):933-35.

17. Bancel B, Patricot LM, Caillon P, Ducerf C, Pouyet M. Hepatic epithelioid hemangioendothelioma. A case with liver transplantation. Review of the literature. Ann Pathol. 1993;13(1):23-28.

18. Kayler LK, Merion RM, Arenas JD, Magee JC, Campbell DA, Rudich SM, et al. Epithelioid haemangioendothelioma of the liver disseminated to the peritoneum treated with liver transplantation and interferon alpha-2B. Transplantation. 2002;74(1):128-30.

19. Alomari AI. The lollipop sign: A new cross-sectional sign of hepatic epithelioid hemangioendothelioma. Eur J Radiol. 2006;59(3):460-64.
20. Frider B, Bruno A, Selser J, Vanesa R, Pascual P, Bistoletti R. Kasabach-Merrit syndrome and adult hepatic epithelioid hemangioendothelioma: an unusual association. J Hepatol. 2005;42(2):282-83.

21. Imanishi H, Kawata M, Yanagihara M, Nakayama N, Sato T, Furukawa Y, et al. Epithelioid hemangioendothelioma of the liver associated with thrombocytopenia and coagulopathy. Hepatogastroenterology. 2002;49(48):1673-675.

22. Galvão FH, Bakonyi-Neto A, Machado MAC, Farias AQ, Mello ES, Diz ME, et al. Interferon alpha-2B and liver resection to treat multifocal hepatic epithelioid hemangioendothelioma: a relevant approach to avoid liver transplantation. Transplant Proc. 2005;37(10):4354-358.

23. Kelleher MB, Iwatsuki S, Sheahan DG. Epithelioid hemangioendothelioma of liver clinicopathological correlation of 10 cases treated by orthotopic liver transplantation. Am J Surg Pathol. 1989;13(12):999-1008.

24. Gelin M, Van de Stadt J, Rickaert F, De Prez C, Levarlet M, Adler M, et al. Epithelioid hemangioendothelioma of the liver following contact with vinyl chloride. Recurrence after orthotopic liver transplantation. J Hepatol. 1989;8(1):99-106.

\section{Abstract}

The authors describe a case of a multicentric epithelioid hemangioendothelioma (EHE) of the liver, a rare hepatic tumor in a patient with nonspecific clinical manifestations, treated initially with resection and ethanol injection. At recurrence, the patient was submitted to radioablation. According to a review of the literature, the treatment of choice is still resection, with or without ablative methods. Liver transplantation appears to be a reasonable therapeutic approach for hepatic EHE when the tumor requires total hepatectomy.

Key words: Epithelioid hemangioendothelioma; Liver; Liver malignancy; Liver transplantation 